\title{
Defects in silicon carbide grown by fluorinated chemical vapor deposition chemistry
}

Pontus Stenberg, Ian Don Booker, Robin Karhu, Henrik Pedersen, ErikJ anzén and Ivan Gueorguiev Ivanov

The self-archived postprint version of this journal article is available at Linköping University Institutional Repository (DiVA):

http:// urn.kb.se/ resolve?urn=urn:nbn:se:liu:diva-147378

N.B.: When citing this work, cite the original publication.

Stenberg, P., Booker, I. D., Karhu, R., Pedersen, H., J anzén, E., Ivanov, I. G., (2018), Defects in silicon carbide grown by fluorinated chemical vapor deposition chemistry, Physica. B, Condensed matter, 535, 44-49. https:// doi.org/ 10.1016/j.physb.2017.06.030

Original publication available at:

https:// doi.org/ 10.1016/j.physb.2017.06.030

Copyright: Elsevier

http://www.elsevier.com/

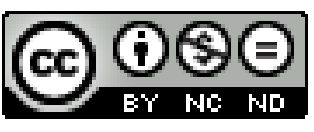




\title{
Defects in silicon carbide grown by fluorinated chemical vapor
}

\section{deposition chemistry}

Pontus Stenberg, Ian D. Booker, Robin Karhu, Henrik Pedersen, Erik Janzén, Ivan G. Ivanov ${ }^{1}$

Department of Physics, Chemistry and Biology, Linköping University, 58183 Linköping, Sweden

\begin{abstract}
Point defects in $n$ - and $p$-type $4 \mathrm{H}$-SiC grown by fluorinated chemical vapor deposition (CVD) have been characterized optically by photoluminescence (PL) and electrically by deep-level transient spectroscopy (DLTS) and minority carrier transient spectroscopy (MCTS). The results are considered in comparison with defects observed in non-fluorinated CVD growth (e.g., using $\mathrm{SiH}_{4}$ instead of $\mathrm{SiF}_{4}$ as silicon precursor), in order to investigate whether specific fluorine-related defects form during the fluorinated CVD growth, which might prohibit the use of fluorinated chemistry for device-manufacturing purposes. Several new peaks identifying new defects appear in the PL of fluorinated-grown samples, which are not commonly observed neither in other halogenated chemistries, nor in the standard CVD chemistry using silane $\left(\mathrm{SiH}_{4}\right)$. However, further investigation is needed in order to determine their origin and whether they are related to incorporation of $\mathrm{F}$ in the $\mathrm{SiC}$ lattice, or not. The electric characterization does not find any new electrically-active defects that can be related to F incorporation. Thus, we find no point defects prohibiting the use of fluorinated chemistry for device-making purposes.
\end{abstract}

${ }^{1}$ Corresponding author, E-mail: ivani@ifm.liu.se 


\section{Introduction}

4H-SiC is a wide indirect bandgap semiconductor of interest for electronic power devices such as unipolar MOSFETs or insulated gate bipolar transistors (IGBTs) [1]. In these and other applications, the chemical vapor deposition (CVD) is the most common technique to grow the material for the active layers. Apart from the traditional CVD growth of $\mathrm{SiC}$ using ethylene $\left(\mathrm{C}_{2} \mathrm{H}_{4}\right)$ and silane $\left(\mathrm{SiH}_{4}\right)$ as carbon and silicon precursors, respectively, the use of chlorine in a SiC CVD process has been found beneficial since chlorine binds stronger to Si than Si to another Si (Si-Cl binding energy is $417 \mathrm{~kJ} / \mathrm{mol}$ compared to Si-Si, $310 \mathrm{~kJ} / \mathrm{mol}$ [2]). Therefore, the presence of $\mathrm{Cl}$ inhibits the homogenous nucleation of $\mathrm{Si}$ in the gas phase and strongly reduces the probability of formation of Si droplets during growth. Also, high growth rates exceeding $100 \mu \mathrm{m} /$ hour have been demonstrated with chlorinated CVD growth [3]. Fluorine is another halogen which forms even stronger than Cl bond to Si (SiF binding energy is $576 \mathrm{~kJ} / \mathrm{mol}$ [2]). Therefore, the fluorinated chemistry is expected to provide advantages similar to the chlorinated one. Due to its high electronegativity, F forms also strong bonds to other elements (e.g., F-Br, F-Ti). This circumstance can potentially alter the unintentional incorporation of foreign elements in the SiC lattice by binding them to $\mathrm{F}$ in the gas phase, before they are incorporated into the epitaxial layer. Due to the strong bonding, however, F itself can be adsorbed on the surface via fluorinated growth species and potentially incorporate into the growing layer [4]. Considering the chlorinated growth, it has been shown by means of secondary-ion mass spectrometry (SIMS) that $\mathrm{Cl}$ incorporates into the grown epilayers in concentration of $\sim 1 \times 10^{14} \mathrm{~cm}^{-3}$. This is however uncertain since the measured value is close to the detection limit [5]. Following this finding, several publications have investigated the possibility of appearance of Cl-related defects or defect complexes in the epitaxial layers [6-9]. However, while $a b$ initio calculations have predicted several defect configurations, such as $\mathrm{Cl}_{\mathrm{C}}-\mathrm{V}_{\mathrm{Si}}$ complexes, $\mathrm{Cl}_{\mathrm{i}}$ interstitials or $\mathrm{Cl}_{\mathrm{C}}$ substitutional defects to be possible, none of these defects has ever been clearly demonstrated experimentally [9].

The incorporation of $\mathrm{F}$ and other atomic species in $\mathrm{SiC}$ grown using fluorinated chemistry has not been studied so far. Intrinsic defects specific to the growth chemistry also may form during growth. Some of the intrinsic and extrinsic defects may be electrically active and have undesired influence on the device performance, because they may trap charge carriers or provide unwanted recombination channels. Therefore, it is necessary to analyze and 
understand which point defects are present in the as-grown epitaxial layers. In all chemistries, the defect formation is influenced by the various growth parameters. Thus, tuning the parameters may be used as a means of controlling the formation of undesired defects, at least to some extent.

As a first step towards better understanding of the defect formation in fluorinated CVD growth, in this work we investigate the photoluminescence (PL) and deep level transient spectroscopy (DLTS) of the samples grown under different conditions using fluorinated chemistry. Both n- and p-type samples are investigated from point of view of possibility to dope (n- and p-type) during fluorinated growth, as well as formation of intrinsic and extrinsic defects in the epitaxial layers. The results are considered in comparison with those obtained from epitaxial layers grown by other chemistries.

\section{Methods}

Growth. A horizontal hot wall chemical vapor deposition system was used for the growth of both n- and p-type doped SiC epitaxial layers. The growth chamber was a graphite susceptor coated with SiC. No rotation of the substrates was available using this susceptor. The growth was performed on substrates of size $16 \mathrm{~mm} \times 16 \mathrm{~mm}$. Standard $4 \mathrm{H}$-SiC substrates with $4^{\circ}$ off-cut angle towards [11 $\left.\overline{2} 0\right]$ were used, with a constant position in the susceptor during growth.

$\mathrm{CH}_{4}$ and $\mathrm{SiF}_{4}$ were used as $\mathrm{C}$ and $\mathrm{Si}$ precursors, respectively. The latter was also the source of $\mathrm{F}$ in the growth chamber. The n- and p-type doping was implemented using during growth controlled flow of $\mathrm{N}_{2}$ and trimethylaluminum (TMAl) for $\mathrm{N}$ (n-type) and $\mathrm{Al}$ (p-type) doping, respectively. $\mathrm{H}_{2}$ purified using palladium membrane was used as carrier gas. The concentration of $\mathrm{SiF}_{4}$ in $\mathrm{H}_{2}$ was $0.125 \%$, thus $\mathrm{Si} / \mathrm{H}_{2}=0.125 \%$. The samples were grown at process temperature of $1600{ }^{\circ} \mathrm{C}$ and process pressure of 100 mbar with the samples positioned $\sim 6 \mathrm{~cm}$ inside the susceptor.

The dopant precursor concentrations were varied in the range 0.1 to 10 for the $\mathrm{N}_{2} / \mathrm{Si}$ ratio and $10^{-5}$ to $10^{-2}$ for the $\mathrm{Al} / \mathrm{Si}$ ratio using $\mathrm{C} / \mathrm{Si}=1$. Also, one additional experiment using $\mathrm{N}_{2} / \mathrm{Si}=100$ was performed. In this latter experiment the amount of $\mathrm{H}_{2}$ carrier gas was reduced to compensate for the high flow of $\mathrm{N}_{2}$. This resulted in 
$\mathrm{Si} / \mathrm{H}_{2}=0.144 \%$ (instead of $\mathrm{Si} / \mathrm{H}_{2}=0.125 \%$ ). However, the $\mathrm{C} / \mathrm{Si}$ ratio and the process pressure and temperature were maintained the same as for the rest of the runs.

The $\mathrm{C} / \mathrm{Si}$ ratio was varied in the range 0.4 to 1.2 in steps of 0.2 , while the dopant-to-silicon ratios were $\mathrm{N}_{2} / \mathrm{Si}=1$ for $\mathrm{N}$-doped samples and $\mathrm{Al} / \mathrm{Si}=10^{-4}$ for Al-doped samples.

The growth time for the nitrogen doped (n-type) samples were $0.5 \mathrm{~h}$ and $2.0 \mathrm{~h}$ for the aluminum doped (p-type) samples. This resulted in thicknesses of the epitaxial layers between 4 and $5 \mu \mathrm{m}$ for the n-type samples, and about $16-20 \mu \mathrm{m}$ for the p-type samples. The growth rate increases with the C/Si ratio approximately linearly from 8 $\mu \mathrm{m} /$ hour at $\mathrm{C} / \mathrm{Si}=0.4$ to $10 \mu \mathrm{m} /$ hour at $\mathrm{C} / \mathrm{Si}=1.2$.

Growth of thicker layers was also attempted. One additional sample grown at temperature of $1650{ }^{\circ} \mathrm{C}$, pressure $100 \mathrm{mbar}, \mathrm{Si} / \mathrm{H}_{2}=0.25 \%$ and $\mathrm{C} / \mathrm{Si}=0.5$. In this case, ethylene $\left(\mathrm{C}_{2} \mathrm{H}_{4}\right)$ was used as carbon precursor. The sample was grown for 5 hours using $\mathrm{N}_{2}$ as a dopant with ratio of $\mathrm{N}_{2} / \mathrm{Si}=0.15$. This particular sample has a thickness of $\sim 100 \mu \mathrm{m}$.

Photoluminescence (PL). The photoluminescence of the samples was measured using $244 \mathrm{~nm}$ excitation from a frequency-doubled Ar ion laser. The detection part consists of a Jobin-Yvon monochromator (HR460) equipped with a grating of $2400 \mathrm{~g} / \mathrm{mm}$ and a CCD camera. This results in a spectral resolution of $\sim 0.5 \AA$ (full width at half maximum, FWHM). The samples were kept in a He bath cryostat at a temperature of $2 \mathrm{~K}$.

Deep level transient spectroscopy (DLTS) and Minority Carrier Transient Spectroscopy (MCTS). Schottky contacts of $1.2 \mathrm{~mm}$ diameter are deposited by thermal evaporation of $\mathrm{Ni}$ (100 $\mathrm{nm}$ thickness). Silver paste on the highly conductive substrate provided the Ohmic contacts. The capacitance transients were measured in the temperature range 77 - $600 \mathrm{~K}$, using a reverse bias of $-10 \mathrm{~V}$ and a superimposed $10 \mathrm{~V}$ filling pulse (up to a total of $0 \mathrm{~V}$ ) of $1 \mathrm{~ms}$ to $10 \mathrm{~ms}$ duration. GS4 correlation function was used to calculate the spectra. [10]

\section{Results and Discussion}


We consider first the doping experiments which aim at obtaining 4H-SiC epilayers of n- and p-type with different controllable doping concentrations. The donor and acceptor doping concentrations of the various samples are assessed by $\mathrm{C}-\mathrm{V}$ measurements, which indicate that in both cases (donors / acceptors) the doping concentration is approximately proportional to the corresponding gas flow of the dopant carrier $\left(\mathrm{N}_{2}\right.$ for $\mathrm{n}$-type doping, and TMAl for p-type). For N-doped uncompensated samples (with negligible acceptor concentration), and if the the donor doping concentration is below $\sim 2 \times 10^{16} \mathrm{~cm}^{-3}$, the concentration can be estimated also from the PL spectra [11].

\subsection{Photoluminescence}

Let us consider now the low-temperature photoluminescence spectra of the n-type samples (nitrogen-doped). Fig. 1(a) shows the PL spectra in the near-band gap region of the samples N-doped to different concentrations, grown with $\mathrm{C} / \mathrm{Si}=1.0$. The spectra exhibit the usual no-phonon lines of the nitrogen-bound exciton (N-BE) denoted by $\mathrm{P}_{0}$ and $\mathrm{Q}_{0}$, as well as the phonon replicas $\mathrm{P}_{\mathrm{xx}}$ (mainly of the $\mathrm{P}_{0}$ line). In addition, the prominent phonon replicas of the free-exciton emission, whenever observable, are denoted with the symbols $\mathrm{I}_{\mathrm{xx}}$. In both cases of free- and bound-exciton replicas, the subscript ' $x x^{\prime}$ ' denotes the approximate phonon energy (in meV) of the phonon involved in the phonon-assisted recombination [12]. The appearance of two no-phonon lines for the N-BE instead of one is due to the existence of two inequivalent lattice sites within the $4 \mathrm{H}-\mathrm{SiC}$ unit cell. Therefore, $\mathrm{N}$ donors occupying the one or the other inequivalent carbon site have quite different properties, in particular, different ionization energies and exciton binding energies, giving rise to two distinct no-phonon lines in the PL spectrum [13]. On the other hand, the ionization energy of the $\mathrm{N}$ donor at the so-called hexagonal lattice site (the other inequivalent one is called "cubic") has approximately two times lower ionization energy than the $\mathrm{N}$ donor at cubic site [13], which leads to 2 times lower exciton binding energy for the former one, in accord with Haynes rule [14]. Therefore, the N-bound exciton at the hexagonal site, which is much more similar to the free exciton than the N-bound exciton at cubic site, exhibits weak no-phonon line (the free-exciton no-phonon line is forbidden by moment conservation law in indirect semiconductors) and strong phonon replicas (which are the only allowed radiative recombination paths if free-exciton recombination is considered). Thus, the N-BE at hexagonal site is responsible for the appearance of the weaker no-phonon $\mathrm{P}_{0}$ line in the spectra and its significantly stronger phonon 
replicas. Conversely, the more strongly bound $\mathrm{N}-\mathrm{BE}$ at cubic site is responsible for the appearance of the substantially stronger no-phonon $\mathrm{Q}_{0}$ line, but its phonon replicas are negligible.
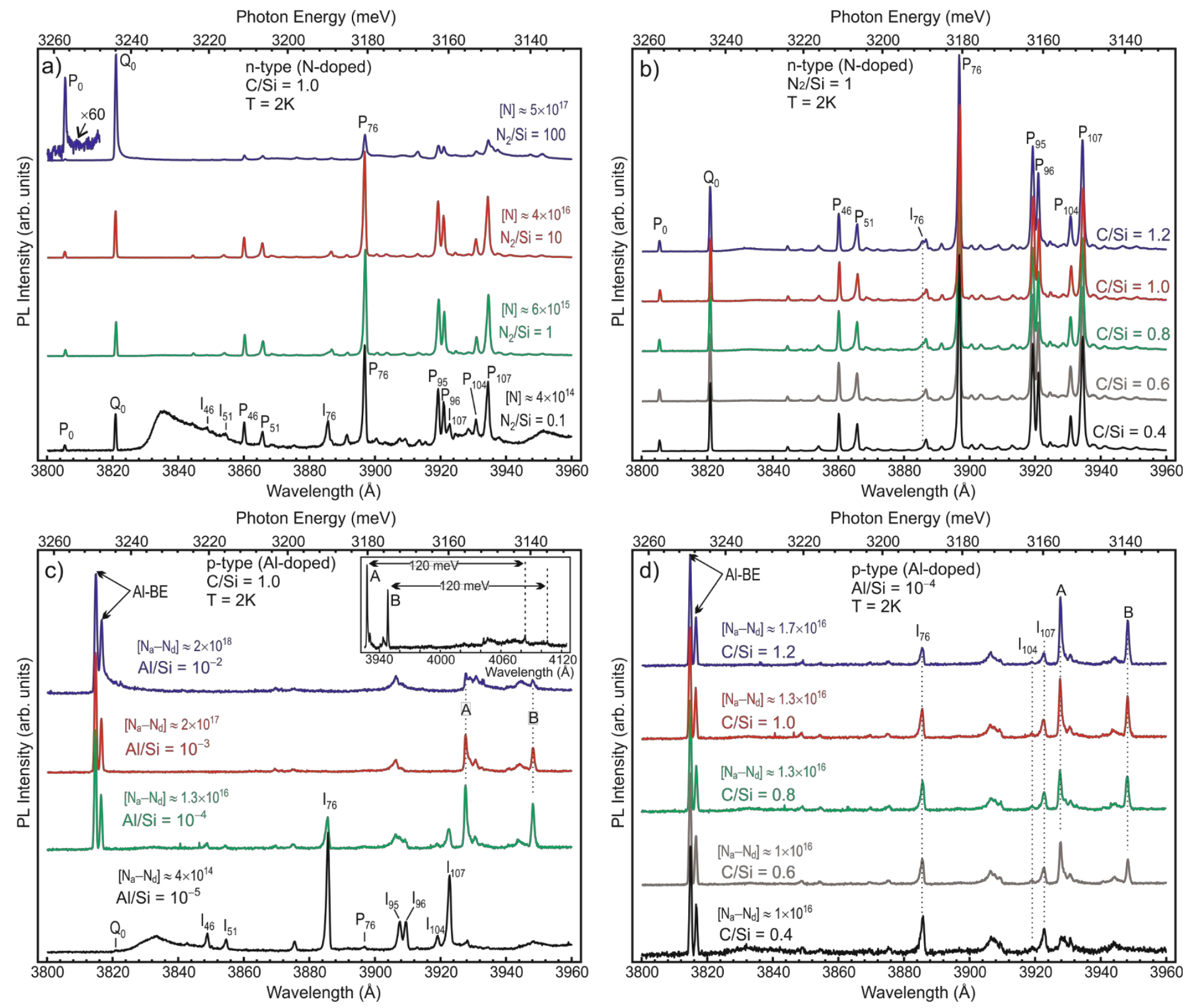

Fig. 1. Normalized low-temperature PL spectra (at $\mathrm{T}=2 \mathrm{~K}$ ) of the samples grown using different growth parameters. (a) n-type, varying $\mathrm{N}_{2} / \mathrm{Si}$ and fixed $\mathrm{C} / \mathrm{Si}=1$. (b) Different $\mathrm{C} / \mathrm{Si}$ ratios at fixed $\mathrm{N}_{2} / \mathrm{Si}=1$. (c) p-type, different $\mathrm{Al} / \mathrm{Si}$ ratios at fixed $\mathrm{C} / \mathrm{Si}=1$. (d) Fixed $\mathrm{Al} / \mathrm{Si}=10^{-4}$, but varying $\mathrm{C} / \mathrm{Si}$. The corresponding parameter changes are denoted for each curve, together with the doping concentration estimated by $\mathrm{C}-\mathrm{V}$ measurements (in $\mathrm{cm}^{-3}$ ). The major no-phonon lines and their replicas are also denoted (see text). The insert in part (c) displays an overview of the defect represented by the A and B lines as discussed in text. 
For the lowest-doped samples (the two bottom curves in Fig. 1(a) ) the free-exciton replica I76, which is strongest in intensity, can be recognized and used for estimation of the N-doping concentration by the method of Ref. [11]. Thus, for the sample with $\mathrm{N}_{2} / \mathrm{Si}=0.1$ we obtain $\mathrm{N}$-donor concentration $[\mathrm{N}]=4 \times 10^{14} \mathrm{~cm}^{-3}$, whereas for the sample with $\mathrm{N}_{2} / \mathrm{Si}=1$ we estimate $[\mathrm{N}] \approx 8 \times 10^{15} \mathrm{~cm}^{-3}$. These values are in excellent agreement with the values of $\mathrm{N}_{\mathrm{D}}-$ $\mathrm{N}_{\mathrm{A}}=4 \times 10^{14} \mathrm{~cm}^{-3}$ and $6.5 \times 10^{15} \mathrm{~cm}^{-3}$, respectively, obtained for the same samples using $\mathrm{C}-\mathrm{V}$ measurements. Here $\mathrm{N}_{\mathrm{D}} \equiv[\mathrm{N}]$ denotes the donor concentration, and $\mathrm{N}_{\mathrm{A}}-$ the acceptor concentration. This agreement between the donor concentrations estimated using the PL spectra (which yields $\mathrm{N}_{\mathrm{D}}$ ) and the C-V measurements (which yields $\mathrm{N}_{\mathrm{D}}-\mathrm{N}_{\mathrm{A}}$ ) suggests that the acceptor concentration is negligible, within the error margin of both methods. We notice also that no new lines which might be associated with F incorporation in the epilayers can be distinguished in this region of the spectra. In other words, the spectra are similar to those observed from epitaxial layers grown using the traditional CVD growth (without presence of halogen elements).

We notice two additional features observable in the spectra in Fig. 1(a). Firstly, the spectrum of the lowest-doped sample (the bottom curve) exhibits significant contribution from broad-band background emission. This is due to (and commonly observed for) highly doped substrates. This background emission abruptly vanishes as the Ndoping level increases, and it is not observable at all in the highly-doped samples $\left(\mathrm{N}_{2} / \mathrm{Si}=10\right.$, and 100$)$. Since the thickness of these samples is the same $(\sim 5 \mu \mathrm{m})$, we consider this feature as an evidence of decreasing free path for the free exciton with increasing doping concentration. Indeed, the free-exciton free path is limited by impurities which can capture (and bind) the free excitons generated by the exciting light. With $244 \mathrm{~nm}$ excitation, the excitons are created within approximately one micrometer from the top surface of the sample. They diffuse further or closer from the surface depending on the concentration of capturing impurities (mostly donors in the case considered). Thus, at low doping concentrations in the epitaxial layer, the excitons may reach the substrate, be captured and recombine there, which is equivalent to indirect excitation of the substrate emission. Thus, the broad-band emission from the substrate in the low-doped layers may serve as an evidence that there are no other exciton-capturing centers in significant concentrations (apart from the intentionally introduced donors) which may serve as radiative or non-radiative inhibitors of the exciton-related luminescence. It is worth noting that substrate emission with $244 \mathrm{~nm}$ excitation can be observed in undoped epitaxial layers with thickness even 
exceeding $50 \mu \mathrm{m}$, according to our own experience, which suggests free-exciton free path of the order of few tens of micrometers in undoped samples with low concentration of exciton-capturing defects.

The second above-mentioned feature concerns the asymmetric broadening of the $\mathrm{Q}_{0}$ and $\mathrm{P}_{0} \mathrm{~N}-\mathrm{BE}$ no-phonon lines at the highest doping level $\left([\mathrm{N}] \approx 5 \times 10^{17} \mathrm{~cm}^{-3}, \mathrm{~N}_{2} / \mathrm{Si}=100\right.$, the top spectrum in Fig. $\left.1(\mathrm{a})\right)$. This broadening is associated not with deteriorated crystalline quality but with the high doping level and is typically observed for highly-doped $4 \mathrm{H}-\mathrm{SiC}$ samples. The effect can be qualitatively understood if one assumes that at high donor concentrations the wave function of an exciton bound to a certain donor will overlap with other donors in the neighborhood. The bound-exciton energy decreases by the energy of interaction with the neighboring donors, which is reflected in emission of photons with slightly lower energies, leading to broadening of the excitonrelated emission lines towards lower photon energies. Another effect can also be envisaged, namely, due to the overlap the exciton bound to a certain donor has finite probability of hopping to another donor in the neighborhood. This hopping migration should be much more pronounced for the excitons bound at the shallower $\mathrm{N}$ donor at hexagonal site (responsible for the $\mathrm{P}_{0}$ line and its replicas) than at the donor at cubic site, due to the more extended exciton wave function associated with the weaker binding, as discussed above. Thus, at high doping concentrations, an exciton initially bound to the $\mathrm{N}$ donor at hexagonal site may migrate and be captured at another $\mathrm{N}$ donor at cubic site. The reverse process (migration away from a cubic site) is much less probable owing to the more localized character of the wave function of an exciton bound at cubic site. This shifts the distribution of excitons bound at hexagonal and cubic sites in favor of the latter, which may explain why at higher doping levels the $\mathrm{P}_{0}$ line and its replicas strongly quench compared to the $\mathrm{Q}_{0}$ line (originating from exciton recombination at cubic site). The quenching of the emission associated with exciton recombination at hexagonal sites ( $\mathrm{P}_{0}$ and its replicas) is well visible in the top curve of Fig. 1(a), which exhibits an order of magnitude smaller ratio of the intensities of $\mathrm{P}_{76} / \mathrm{Q}_{0}$ compared to the rest of the spectra.

Fig. 1(b) displays the spectra of the $\mathrm{N}$-doped samples grown with $\mathrm{N}_{2} / \mathrm{Si}=1$ and various $\mathrm{C} / \mathrm{Si}$ ratios. The spectra are very similar despite the relatively large range of variation of $\mathrm{C} / \mathrm{Si}$ from 0.4 to 1.2 . The change in the $\mathrm{C} / \mathrm{Si}$ ratio is accompanied with weak almost linear with $\mathrm{C} / \mathrm{Si}$ change of the growth rate from $\sim 8 \mu \mathrm{m} / \mathrm{h}$ at $\mathrm{C} / \mathrm{Si}=0.4$ to $\sim 10$ $\mu \mathrm{m} / \mathrm{h}$ at $\mathrm{C} / \mathrm{Si}=1.2$. The $\mathrm{N}$-doping concentration $[\mathrm{N}]$ also changes from $\sim 2 \times 10^{17} \mathrm{~cm}^{-3}$ at $\mathrm{C} / \mathrm{Si}=0.4$ to $\sim 3 \times 10^{16}$ 
$\mathrm{cm}^{-3}$ at $\mathrm{C} / \mathrm{Si}=1.2$, as estimated using $\mathrm{C}-\mathrm{V}$ measurements. These changes are in good agreement with the site competition mechanism proposed by Larkin et al. [15], according to which nitrogen and carbon compete for the same lattice sites (C sites). Therefore, the growth with C-rich conditions (high C/Si ratio) results in lower $\mathrm{N}$ doping, and vice versa. The changes in [N] are also observable in the PL spectra in Fig. 1(b); the dotted line denotes the position of the free-exciton replica $\mathrm{I}_{76}$, which is well visible in the top spectrum $(\mathrm{C} / \mathrm{Si}=1.2)$, but gradually vanishes with decreasing $\mathrm{C} / \mathrm{Si}$ and is absent in the bottom curve $(\mathrm{C} / \mathrm{Si}=0.4)$.

We consider now the p-type doping experiments. The PL spectra obtained from the samples grown with C/Si $=$ 1.0 and different Al-doping (different $\mathrm{Al} / \mathrm{Si}$ ratios) are displayed in Fig. 1(c). The figure displays also the net concentration of acceptors minus donors $\left[\mathrm{N}_{\mathrm{a}}-\mathrm{N}_{\mathrm{d}}\right]$ for each sample obtained from $\mathrm{C}-\mathrm{V}$ measurements, indicating nearly linear growth of the doping concentration with the $\mathrm{Al} / \mathrm{Si}$ ratio for $\mathrm{Al} / \mathrm{Si} \geq 10^{-4}$. Also, the Al-related bound exciton lines (Al-BE, cf. Clemen et al. [16]) are dominating the spectra of all samples with $\mathrm{Al} / \mathrm{Si} \geq 10^{-4}$. The sample grown with $\mathrm{Al} / \mathrm{Si}=10^{-5}$, however, deserves special attention because, apart from the broad bands due to the substrate, the only prominent contribution in the spectrum of this sample is due to the free-exciton emission lines. The Al-BE lines are not observed at all, and the otherwise strong $\mathrm{N}-\mathrm{BE}$ lines $\mathrm{Q}_{0}$ and $\mathrm{P}_{76}$ appear only as weak bumps, as shown in this spectrum. This peculiar situation may indicate nearly perfect compensation of donors and acceptors in this sample, leading to ionized donors and acceptors with negligible concentrations of the neutral species. Since the ionized donors and acceptors in $4 \mathrm{H}-\mathrm{SiC}$ cannot bind excitons due to the specific ratio of the electron and hole effective masses, the free-exciton emission prevails. The substrate emission is also excited indirectly in this sample, as discussed earlier. The free-exciton emission (e.g., $\left.I_{76}\right)$ is still discernible in the sample with $\mathrm{Al} / \mathrm{Si}=10^{-4}$, but vanishes with increasing Al-doping concentration, as expected. Also, the Al$\mathrm{BE}$ lines of the highest-doped sample $\left(\mathrm{Al} / \mathrm{Si}=10^{-2}\right)$ exhibit asymmetric broadening, which can be understood on the same ground as that for the N-BE already discussed.

There are almost no distinct phonon replicas of the no-phonon Al-BE lines (the two no-phonon lines are associated with $\mathrm{Al}$ occupying the two inequivalent $\mathrm{Si}$ sites in the $4 \mathrm{H}-\mathrm{SiC}$ unit cell). However, two prominent sharp lines are seen to appear at 3928 and $3948 \AA$ (3156 and 3139 meV, respectively), denoted as A and B in Fig. 1(c,d). Both lines exhibit a weak phonon-assisted spectrum as illustrated in the insert of Fig. 1(c), which consists 
of continuous emission typical for deep centers, which ends abruptly with a cusp at about 120 meV above the respective line (see the insert,120 $\mathrm{meV}$ is the maximum phonon energy in $4 \mathrm{H}-\mathrm{SiC}$ ). The $\mathrm{A}$ and $\mathrm{B}$ lines are typically not observed in Al-doped $4 \mathrm{H}-\mathrm{SiC}$. The existence of two lines each of which presents also a phonon spectrum suggests that they might be associated with the same defect in two inequivalent configurations within the unit cell of $4 \mathrm{H}-\mathrm{SiC}$. The origin of this defect is not understood at present, but it might be coupled to the incorporation of $\mathrm{F}$ in the $\mathrm{SiC}$ lattice. It may be noticed further that the intensity ratio of the $\mathrm{A}$ and $\mathrm{B}$ lines is nearly constant, and both lines vanish at high Al doping concentration (cf. Fig. 1(c), top curve).

Fig. 1(d) displays the spectra of the samples grown with constant $\mathrm{Al} / \mathrm{Si}$ and varying $\mathrm{C} / \mathrm{Si}$ ratio from 0.4 to 1.2 , similar to the case of n-type doping considered above. Considering again the A and B lines, we notice that their intensity decreases with decreasing $\mathrm{C} / \mathrm{Si}$ ratio and nearly vanishes at the lowest $\mathrm{C} / \mathrm{Si}=0.4$ (Fig. 1 (d)). This might indicate that the defect responsible for the A and B lines is due to atomic species competing with Si for the Si site. Therefore, the A and B formation is promoted in C-rich growth conditions. Thus the defect responsible for the A and B lines may be tentatively assigned to an unknown foreign atom (or complex) occupying the Si site, although further experimental evidence using other techniques (e.g., electron spin resonance) is required to confirm or reject this assignment.

As seen from Fig. 1(d), there is a marginal increase in $\left[N_{a}-N_{d}\right]$ with increasing C/Si ratio, but this increase is less than a factor of two, from $1 \times 10^{16}$ to $1.7 \times 10^{16} \mathrm{~cm}^{-3}$. The intensity ratio of the free-exciton line $\mathrm{I}_{76}$ visible in all spectra to the Al-BE lines exhibits some variations which are not very well correlated with $\left[\mathrm{N}_{\mathrm{a}}-\mathrm{N}_{\mathrm{d}}\right]$ measured by $\mathrm{C}-\mathrm{V}$. This is probably due to different compensation levels because of different $\mathrm{N}$-concentration, however, the N-BE lines are not detectable in any of the p-type samples presented in Fig. 1(c,d).

The photoluminescence of all samples was measured in the range $380-945 \mathrm{~nm}(\sim 3.26-1.31 \mathrm{eV})$. Apart from the near-band gap region already considered, the only contribution to the rest of the spectrum is the appearance of the titanium-related emission in some of the samples. The spectra of p-type and n-type samples with the most prominent Ti emission are displayed in Fig. 2. The intensities of the Ti lines $\mathrm{B}_{0}$ and its local phonon mode $\mathrm{B}_{90}$ are of the order of 10 - 100 times weaker than the corresponding bandgap emission for the n- and p-type samples, respectively. Ti is common contaminant in CVD epitaxy, probably emitted from the susceptor, but we notice the 
complete absence of the so-called $A_{0}$ line [17] and its phonon replica $A_{90}$. The absence of this line usually associated with one of the two inequivalent sites of Ti substituting Si is not specific to the fluorinated growth. According to ours (and other groups) unpublished results the $A_{0}$ line is not always present in the spectrum also in samples using conventional growth without presence of halogens. However, the reason for its absence in many samples is not understood at present.

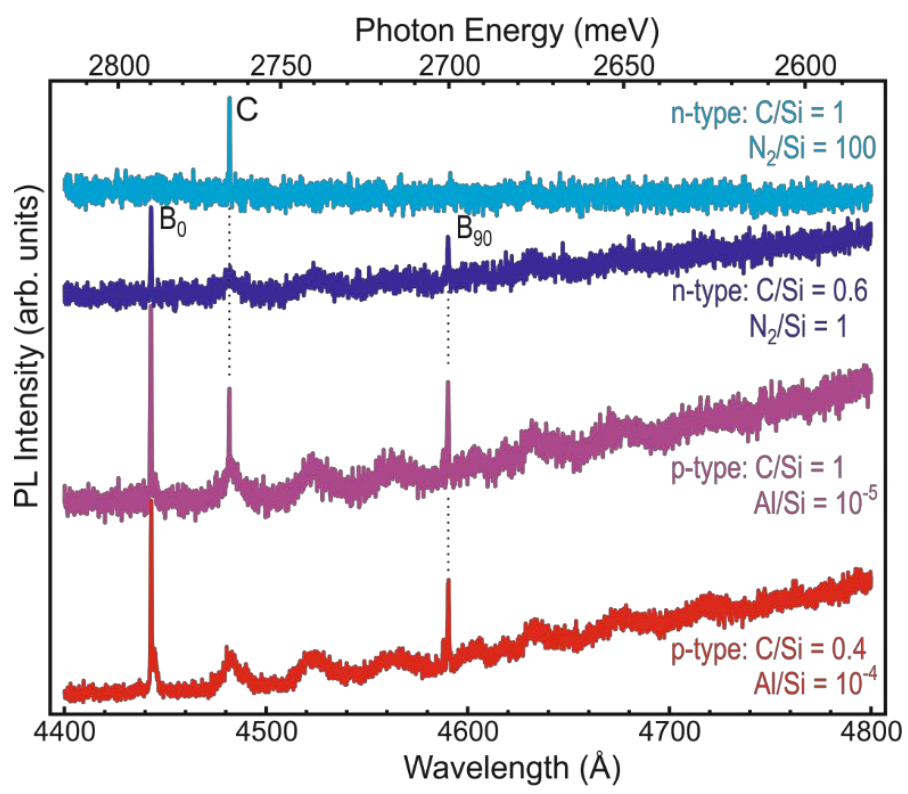

Fig 2. Low-temperature PL spectra $(T=2 \mathrm{~K})$ of selected samples of n-type and p-type which exhibit the strongest emission of the Ti-related defect ( $\mathrm{Ti}$ on the Si sites) and an unknown sharp line (denoted $\mathrm{C}$ ) which belongs to an unidentified defect. The samples, the near-band gap emission of which is displayed in Fig. (1), are identified (and labeled) by the corresponding growth parameters.

The spectra in Fig. 2 exhibit one more sharp line of unknown origin, which is not commonly observed and might be specific to the fluorinated chemistry. This line appearing at $4482 \AA$ (2765 meV) and denoted as C in Fig. 2 appears in almost all (but not all) n-type samples, as illustrated by the top two curves, and seems to be independent of the presence or absence of the Ti spectrum. It appears also in p-type, but only in one of the samples - the one that has no contribution from either Al-BE or N-BE (the bottom curve in Fig. 1(c)). Further investigation is needed to clarify the nature of the defect responsible for the appearance of this line.

We turn now to discussion of the DLTS and MCTS results. 


\subsection{Deep level transient spectroscopy}

Spectra were collected from epitaxial layers grown using standard, chlorinated, brominated and fluorinated chemistry, Fig. $3[18,19]$. The detection limit in these epitaxial layers, determined from the background doping, was $1 \times 10^{11} \mathrm{~cm}^{-3}$, except for the chlorinated epitaxial layers, where it extended down to $1 \times 10^{10} \mathrm{~cm}^{-3}$. The same electrically active deep levels appear in all epitaxial layers independent of used chemistry, except for the A(1) and A(2) levels, discussed later. All deep level parameters are given in Table 1.

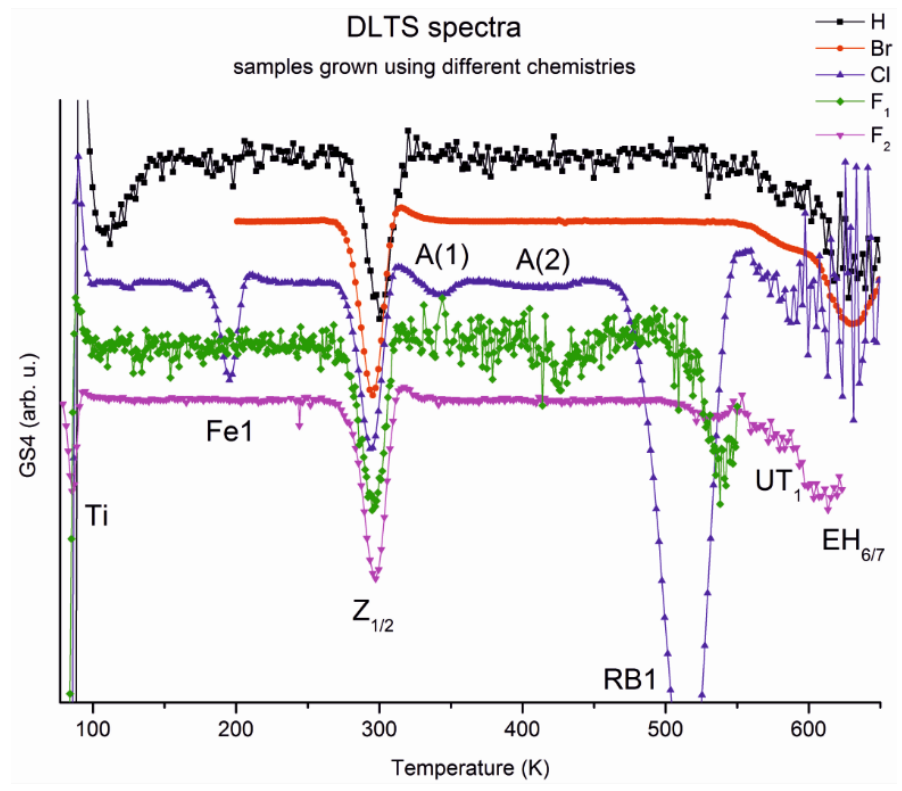

Figure 3. DLTS spectra for samples grown using standard $(\mathrm{H})$, brominated $(\mathrm{Br})[19]$, chlorinated $(\mathrm{Cl})$ and fluorinated chemistry represented by two samples $\left(\mathrm{F}_{(1)}-5 \mu \mathrm{m}\right.$ thick, and $\mathrm{F}_{(2)}-100 \mu \mathrm{m}$ thick). 
Table 1: Approximate thermal activation energies $\left(E_{a}\right)$ and temperature-independent capture cross sections $\left(\sigma_{e}\right)$ of deep levels found in epilayers grown using standard, chlorinated, brominated and fluorinated precursor chemistries. For simplicity, the levels were fitted assuming single electron emission processes.

\begin{tabular}{|l|l|l|l|}
\hline Level & $E_{\mathrm{a}}[\mathrm{eV}]$ & $\sigma_{\mathrm{e}}\left[\mathrm{cm}^{2}\right]$ & Comment \\
\hline $\mathrm{Ti}$ & 0.16 & $5 \times 10^{-15}$ & All chemistries \\
\hline Fe1 & 0.39 & $1 \times 10^{-15}$ & Chlorinated precursors, Fe-related \\
\hline $\mathrm{Z}_{1 / 2}$ & 0.69 & $9 \times 10^{-15}$ & All chemistries, related to carbon vacancy $\left.\mathrm{V}_{\mathrm{C}}\right)$ \\
\hline $\mathrm{A}(1)$ & 0.71 & $7 \times 10^{-16}$ & Chlorinated precursors, possibly Cl-related \\
\hline $\mathrm{A}(2)$ & 0.9 & $6 \times 10^{-16}$ & Chlorinated precursors, possibly Cl-related \\
\hline RB1 (RD $1 / 2)$ & 1.05 & $2 \times 10^{-16}$ & All chemistries, defect-complex including $\mathrm{V}_{\mathrm{C}}$ \\
\hline $\mathrm{UT}_{1}$ & 1.4 & $1 \times 10^{-13}$ & All chemistries, possibly complex including C \\
\hline $\mathrm{EH}_{6 / 7}$ & 1.48 & $1 \times 10^{-14}$ & All chemistries, V -related \\
\hline
\end{tabular}

Common to all epitaxial layers were the carbon vacancy $\left(\mathrm{V}_{\mathrm{C}}\right)$ related $\mathrm{Z}_{1 / 2}$ and $\mathrm{EH}_{6 / 7}$ deep level signals, with the as-grown $\mathrm{V}_{\mathrm{C}}$-concentration between mid- $10^{11} \mathrm{~cm}^{-3}$ to mid- $10^{13} \mathrm{~cm}^{-3}$. These deep levels also constitute the main point defect-related non-radiative recombination pathway for charge carriers. Since different reactors were used for the experiments and growth conditions for the layers varied widely, a direct comparison of concentrations between chemistries is not possible.

A secondary, weaker recombination center is the RB1 deep level, which occurs together with the Fe-related Fe1 level in most of our epitaxial layers grown with chlorinated chemistry. While RB1 appears to be an intrinsic defect which may be removed by oxidation, its formation scales sub-linearly with the concentration of 
incorporated Fe [18]. The level appears to be identical to the known intrinsic $\mathrm{RD}_{1 / 2}$ level, and also appears in some brominated and fluorinated epitaxial layers which lack any detectable Fe-contamination [20].

The most common electron trapping deep levels which was detected in epitaxial layers grown by all chemistries are related to $\mathrm{Ti}$, a transition metal impurity originating from the graphite insulation. Another, less common trap found in some epitaxial layers independent of the chemistry used is the $\mathrm{UT}_{1}$ level, which appears to have a very large electron capture cross section and is possibly related to a carbon cluster defect [21].

The only unique deep levels are A(1) and A(2), which were only detected in chlorinated epitaxial layers in very low concentrations of below mid- $10^{11} \mathrm{~cm}^{-3}$ (the concentration ratio is $\sim 2: 1$ ). One explanation for this may be the slightly higher detection limit in the non-chlorinated epitaxial layers. Another explanation may be that these deep levels are related to Cl-incorporation, as their positions in the spectrum correspond to that of the levels Ci4 and Ci5, simulated using their literature parameters [9]. These levels, which were first found in Cl-implanted epitaxial layers, have been speculated to originate from Cl-containing defect complexes. Their density in our as-grown epitaxial layers is well below the previously determined concentration of $\mathrm{Cl}$ incorporated during growth [5]. No similar unique deep levels have been found in the epilayers grown either using brominated or fluorinated chemistry.

\section{Conclusions}

SiC grown using a fluorinated CVD chemistry and investigated using PL, DLTS and MCTS shows properties very similar to those of samples grown with other chemistries. Fluorinated chemistry is therefore a possible route to grow epitaxial layers for $\mathrm{SiC}$ devices. However, we find also some minor features in the photoluminescence, which might be specific to the F-chemistry, and which need further investigation for understanding their nature.

Funding: This work was supported by the Knut \& Alice Wallenberg Foundation (KAW) project “Isotopic Control for Ultimate Material Properties”, the SSF project “SiC - the Material for Energy-Saving Power Electronics” and the VR Grant Nr. 2016-05362. 


\section{References}

1. J. A. Cooper, A. Agarwal, Proc. IEEE 90, 956 (2002).

2. Y.-R. Luo, J.-P. Cheng, Bond Dissociation Energies, in CRC Handbook of Chemistry and Physics, pp. 9-65 - 9-70 (96 ${ }^{\text {th }}$ Edition, Ed. W.M. Haynes,CRC Press, Boca Raton - London - New York, 20152016).

3. H. Pedersen, S. Leone, O. Kordina, A. Henry, S. Nishizawa, Y. Koshka, E. Janzén, Chem. Rev. 112, 2434 (2012).

4. P. Stenberg, Fluorinated SiC CVD, PhD thesis (Linköping University 2017).

5. H. Pedersen, S. Leone, A. Henry, V. Darakchieva, P. Carlsson, A. Gällström, E. Janzén, phys. stat. sol (RRL) 2, 188 (2008).

6. F.C. Beyer, H. Pedersen, A. Henry, E. Janzén, Mater. Sci. Forum Vols. 615-617, 373 (2009).

7. G Alfieri and T Kimoto, J. Phys.: Condens. Matter 23, 415802 (2011).

8. G. Alfieri and T. Kimoto, J. Appl. Phys. 111, 103705 (2012).

9. G. Alfieri and T. Kimoto, J. Appl. Phys. 112, 063717 (2012).

10. A. A. Istratov, Rev. Sci. Instr. 68, 3861 (1997).

11. I. G. Ivanov, C. Hallin, A. Henry, O. Kordina, E. Janzén, J. Appl. Phys. 80, 3504 (1996).

12. I. G. Ivanov, U. Lindefelt, A. Henry, O. Kordina, C. Hallin, M. Aroyo, T. Egilsson, E. Janzén, Phys. Rev. B 58, 13634 (1998).

13. I. G. Ivanov, A. Henry, E. Janzén, Phys. Rev. B 71, 241201(R) (2005).

14. J. R. Haynes, Phys. Rev. Lett. 4, 361 (1960). 
15. D. J. Larkin, P. G. Neudeck, J. A. Powell, L. G. Matus, Appl. Phys. Lett. 65, 1659 (1994).

16. L.L. Clemen, R.P. Devaty, W.J. Choyke, J.A. Powell, D.J. Larkin, J.A. Edmond, A.A. Burk, in The Proceedings of the 5th Conference on Silicon Carbide and Related Materials (1-3 November 1993, Ed. M.G. Spencer, R.P. Devaty, J.A. Edmond, M. Asif Khan, R. Kaplan, and M. Rahman), IOP Conf. Proc. No. 137 (Institute of Physics, London, 1994), p. 297.

17. L. Patrick, W. J. Choyke, Phys. Rev. B 10, 5091 (1974).

18. I. D. Booker, J. Hassan, L. Lilja, F. C. Beyer, R. Karhu, J. P. Bergman†, Ö. Danielsson, O. Kordina, E. Ö. Sveinbjörnsson, E. Janzén, Cryst. Growth Des. 14, 4104 (2014).

19. M. Yazdanfar, Ö. Danielsson, E. Kalered, P. Sukkaew, O. Kordina, D. Nilsson, I. G. Ivanov, L. Ojamäe, E. Janzén, H. Pedersen, Chem. Mater. 27, 793 (2015).

20. T. Dalibor, G. Pensl, H. Matsunami, T. Kimoto, W. J. Choyke, A. Schöner, N. Nordell, phys. status solidi (a) 162, 199 (1997).

21. K. Danno, T. Hori, T. Kimoto, J. Appl. Phys. 101, 053709 (2007). 